\title{
Outcomes and clinical features of leptomeningeal carcinomatosis: A single center experience
}

\author{
Derya KIVRAK SALIM $®$, Arif Hakan ONDER
}

\begin{abstract}
Objective: The aim of this study was to identify the clinical features and prognostic factors of cancer patients with leptomeningeal carcinomatosis (LMC) in a single center.

Patients and Methods: Patients 18 and older who had LMC diagnosis between 2013 and 2018 at Medical Oncology Department, Antalya Education and Research Hospital, Health Sciences University were included into the study. Their clinical features, treatment approaches, overall survival, survival after LMC diagnosis and prognostic factors on survival were retrospectively investigated.

Results: Sixteen solid cancer patients included in the study. The median time from primary tumor diagnosis to LMC diagnosis was 6 months (range, 1-180 months). The median time from LMC diagnosis to death was 1.5 months (range, 1-14 months). The median overall survival for the entire population was 11 months (95\%CI 5.7-16.3). Age $(p=0.6)$, gender $(p=0.51)$, metastases areas (for liver metastases $\mathrm{p}=0.95$, for lung metastases $\mathrm{p}=0.26$, for bone metastases $\mathrm{p}=0.82$ ), The Eastern Cooperative Oncology Group Performance Status ( ECOG PS) $(\mathrm{p}=0.18$ ), treatment type of LMC (only radiation therapy (RT) $\mathrm{p}=0.33$; RT followed by intrathecal methotrexate (IT MTX) $(p=0.35)$, RT type $(p=0.76)$ and time from primary tumor diagnosis to LMC diagnosis $(p=0.50)$ did not show prognostic effect on overall survival after LMC diagnosis.

Conclusion: Overall survival after LMC diagnosis is too short to see the effect of treatment modalities. Our study did not find any favorable or unfavorable prognostic factor on survival after LMC diagnosis.
\end{abstract}

Keywords: Leptomeningeal carcinomatosis, Survival, Prognostic factors

Derya Kivrak Salim ( $\varangle$ ), Arif Hakan Onder

Department of Medical Oncology, Antalya Education and Research

Hospital, Health Sciences University, Antalya, Turkey.

e-mail:deryakivrak@gmail.com

\section{Introduction}

Leptomeningeal carcinomatosis (LMC) is a fatal complication of systemic cancer in which cancer cells seed through meninx and cerebrospinal fluid (CSF). Incidence of LMC in solid cancers vary from $1 \%$ to $10 \%[1,2]$. LMC is mostly seen in breast cancer patients [3]. It shows a poor prognosis with median survival of 14 weeks for lung cancer [1], 7 weeks for gastrointestinal cancers [2], 12 weeks for breast cancer [4]. Overall survival for LMC of both solid and hematopoietic cancers was 12 weeks [4]. Standard diagnostic approach is the sampling of CSF and demonstration of cancer cells. But the sensitivity of sampling is poor due to high false negative result rates and only $55 \%$ of patients with LMC has positive cytology at initial examination [5]. Technical development in neuroradiology resulted in increased LMC diagnosis rates with MRI by finding meningeal enhancement [3]. The treatment options of LCM are mainly the same for all types of cancer but incidence and prognosis change according to histology of primary cancer. The survival differences cannot be explained or predicted with the present data. Mostly symptomatic treatments for headache, nausea, vomiting and back pain are performed. Intrathecal (IT) chemotherapy, whole brain radiotherapy (WBRT) and/or site specific radiotherapy are more specific therapeutic options [6,7]. Thiotepa, methotrexate and cytarabine are commonly used agents for IT route [7]. Despite the fact that central nervous system (CNS) is a privileged site and blood-brain-barrier limits the influx of cytotoxic drugs, systemic treatment in breast cancer and lung cancer patients with LMC showed clinical benefit in $15.8 \%$ of patients [8]. There are limiting retrospective studies evaluating survivals and prognostic factors in LMC patients. The aim of this study was to identify the clinical features and prognostic factors of cancer patients with LMC from a single center. 


\section{Patients and Methods}

\section{Study Design and Patients}

Cancer patients aged $\geq 18$ year-old with cytologically proven LMC between 2013 and 2018 at Medical Oncology Department, Antalya Education and Research Hospital, Health Sciences University were included into the study. Medical records of 16 patients were retrieved and retrospectively analyzed. The study was approved by the Health Sciences University, Antalya Education and Research Hospital Clinical Research Ethics Committee on $21^{\text {st }}$ February , 2019 (approval number:2019-045, 6/2). Patients with primary CNS tumors and hematologic malignancies were excluded. Clinical features of LMC patients with disseminated solid cancers and their prognostic factors for survival were retrospectively investigated.

\section{Statistical Methods}

Statistical analysis was made using IBM SPSS Statistics for Windows, Version 23.0 (IBM Corp., Armonk, NY). Descriptive analyses were presented using median (min$\max )$ or $\mathrm{n}(\%)$, where appropriate. Overall survival (OS) was estimated using the Kaplan-Meier method. The log-rank test was used to compare survival differences. A univariate Cox proportional hazards regression model was used to identify prognostic factors. Hazard ratio, with corresponding 95\% confidence intervals ( $95 \% \mathrm{CIs}$ ), was reported. A p-value of less than 0.05 was considered statistically significant.

\section{Results}

Sixteen patients who had diagnosis of LMC at a single center between 2013 and 2018 were included into the study. Median age of LMC onset was 53 years (range, 2372 years). There were 7 male and 9 female patients. The Eastern Cooperative Oncology Group Performance Status (ECOG PS) was $<2$ in 3 patients $(18.8 \%$ ) while it was $\geq 2$ in 13 patients $(81.2 \%)$ for LMC. Clinical demographics, primary tumor histology and treatment details are presented in Table I. The median time from primary tumor diagnosis to LMC diagnosis (TPM) was 6 months (range, 1-180 months). The most common clinical presentations were cerebral symptoms such as headache $(68.8 \%)$ and mental confusion $(56.3 \%)$. Presenting symptoms were presented in Table I and Figure 1. The median time from LMC diagnosis to death (TMD) was 1.5 months (range,
1-14 months). The median overall survival (m OS) for the entire population was 11 months $(95 \% \mathrm{CI}$ 5.7-16.3) (Figure 2).

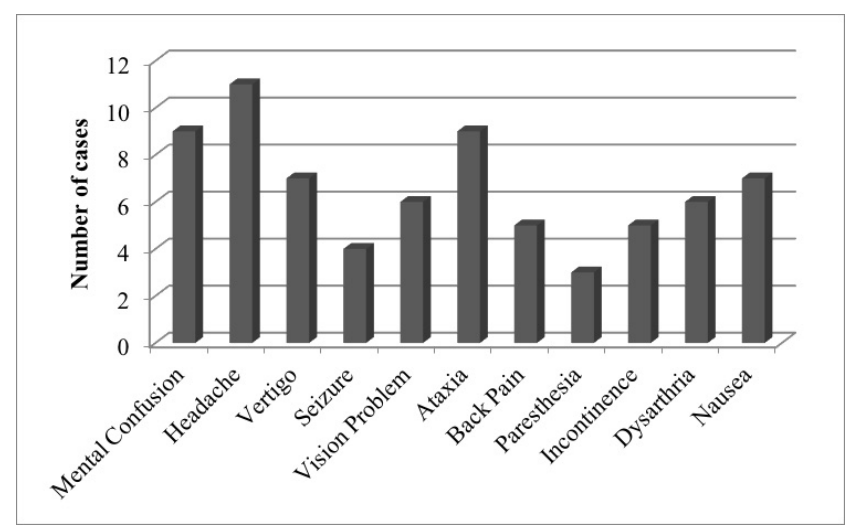

Figure 1. Presenting symptoms of LMC

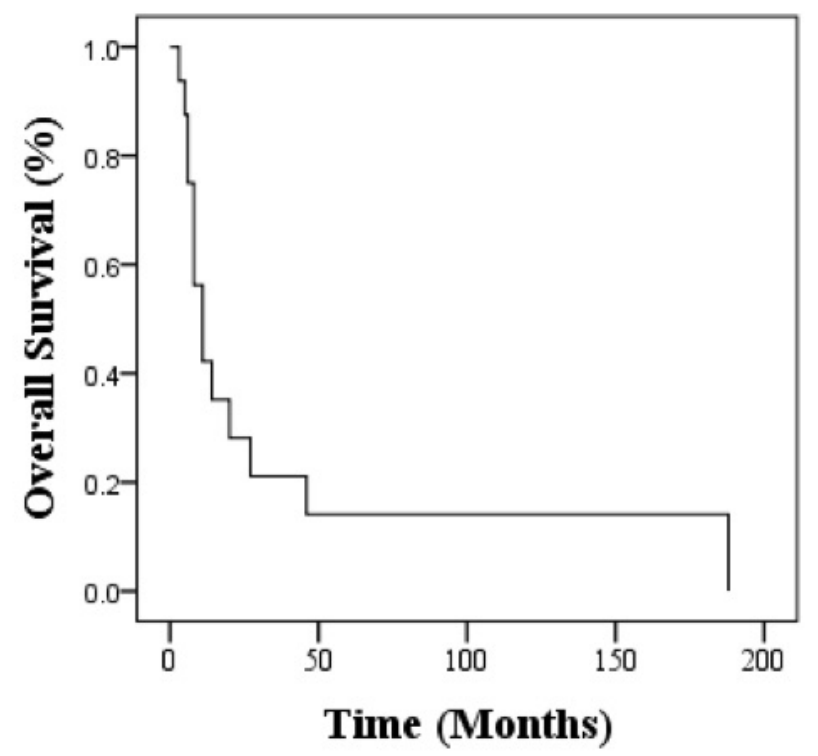

Figure 2. Median OS after primary cancer diagnosis in general population was 11 months $(95 \% \mathrm{CI}, 5.7-16.3)$

After LMC diagnosis only two patients received best supportive care, 8 patients $(50 \%)$ had only radiotherapy (RT) and 6 patients had sequential RT and intrathecal methotrexate (MTX) (Table I). There were no OS differences between cranial RT and craniospinal RT groups ( $\mathrm{p}=0.71)$ (Figure 3), also IT MTX treatment did not prolong the survival after LMC treatment $(\mathrm{p}=0.75)$ (Figure 4). Age (HR, 0.988; 95\%CI, 0.947-1.032, $\mathrm{p}=0.6)$, gender (HR1.459; 95\%CI, 0.479-4.444, $\mathrm{p}=0.51$ ), metastatic areas (for liver metastases $\mathrm{p}=0.95$, for lung 
metastases $\mathrm{p}=0.26$, for bone metastases $\mathrm{p}=0.82$ ) (Table II), ECOG PS at LMC diagnosis (HR,2.976; 95\%CI, 0.602$14.718, \mathrm{p}=0.18$ ), presence of synchronous parenchymal mass (HR, $0.858 ; 95 \% \mathrm{CI}, 0.262-2.810, \mathrm{p}=0.80)$, treatment options for LMC (only RT $\mathrm{p}=0.33$; RT and IT MTX $\mathrm{p}=0.35)$, RT type $(\mathrm{p}=0.76)$ and time from primary tumor diagnosis to LMC diagnosis (TPM) (HR, 1.458; 95\% CI, 0.484-4.392, $\mathrm{p}=0.50$ ) did not show prognostic effect on overall survival after LMC diagnosis.

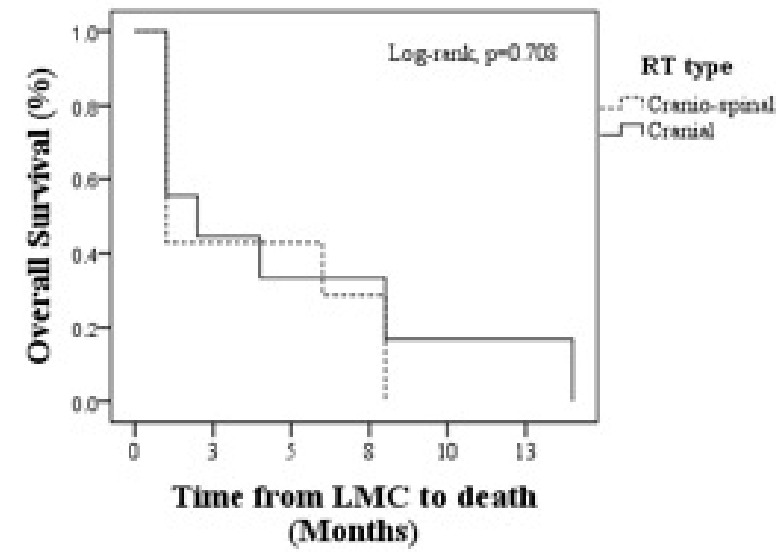

Figure 3. OS with craniospinal RT vs cranial RT

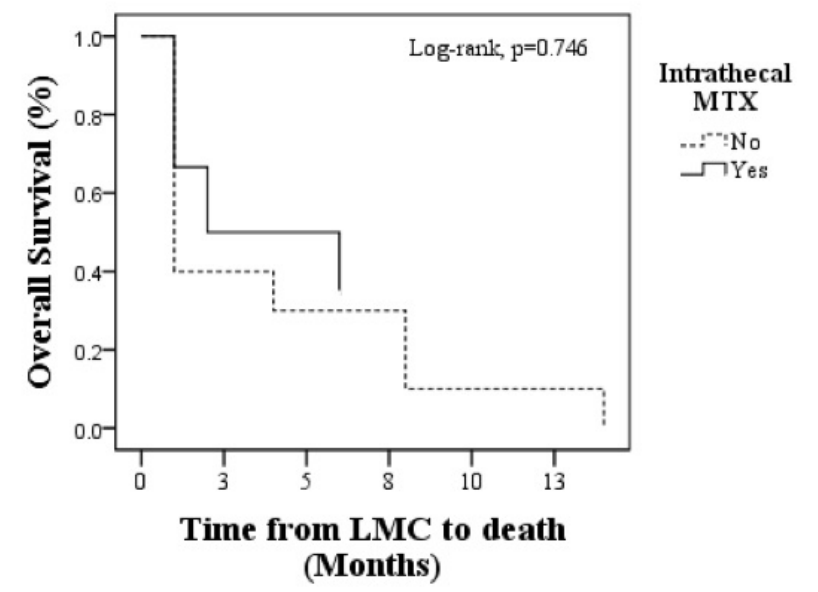

Figure 4. OS with implication of intrathecal MTX
Table I. Clinical Demographics

\begin{tabular}{|c|c|}
\hline & $\mathrm{n}: 16$ \\
\hline Primary Tumor, n(\%) & \\
\hline Lung cancer & $6(37.5)$ \\
\hline Breast cancer & $3(18.8)$ \\
\hline Colon cancer & $1(6.3)$ \\
\hline Medullary thyroid cancer & $1(6.3)$ \\
\hline Gastric cancer & $2(12.5)$ \\
\hline Endometrial cancer & $1(6.3)$ \\
\hline Pancreas cancer & $1(6.3)$ \\
\hline Nonseminomatous testis cancer & $1(6.3)$ \\
\hline Metastatic disease, $n(\%)$ & \\
\hline Liver metastases & $4(25)$ \\
\hline Lung metastases & $9(56.3)$ \\
\hline Bone metastases & $9(56.3)$ \\
\hline RT Type & \\
\hline Cranio-spinal & $5(31.25)$ \\
\hline Cranial & $9(56.25)$ \\
\hline LMC treatment & \\
\hline No Treatment & $2(12.5)$ \\
\hline RT & $8(50)$ \\
\hline RT+Intrathecal MTX & $6(37.5)$ \\
\hline Parenchymal Mass, n(\%) & \\
\hline Yes & $11(68.8)$ \\
\hline No & $5(31.3)$ \\
\hline Stage at diagnosis, $n(\%)$ & \\
\hline Stage II & $3(18.8)$ \\
\hline Stage III & $2(12.5)$ \\
\hline Stage IV & $11(68.8)$ \\
\hline MRI Findings at LMC diagnosis, n (\%) & \\
\hline Normal & $5(31.3)$ \\
\hline Intracranial mass & $3(18.8)$ \\
\hline Leptomeningeal thickening & $6(37.5)$ \\
\hline Ventricular dilatation & $2(12.5)$ \\
\hline Presenting Symptoms of LMC, n(\%) & \\
\hline Mental confusion & $9(56.3)$ \\
\hline Headache & $11(68.8)$ \\
\hline Vertigo & $7(43.8)$ \\
\hline Seizure & $4(25)$ \\
\hline Vision Problems & $6(37.5)$ \\
\hline Ataxia & $9(56.3)$ \\
\hline Back Pain & $5(31.3)$ \\
\hline Paresthesia & $3(18.8)$ \\
\hline Incontinence & $5(31.3)$ \\
\hline Dysarthria & $6(37.5)$ \\
\hline Nausea & $7(43.8)$ \\
\hline
\end{tabular}

RT. Radiothrapy, LMC: Leptomeningeal carcinomatosis, MTX: Methatrexate 
Table II. Prognostic factors affecting OS after LMC diagnosis

\begin{tabular}{lll}
\hline Variables & HR $(95 \% \mathrm{CI})$ & $\mathrm{p}$ \\
\hline Age & $0.988(0.947-1.032)$ & 0.598 \\
\hline Gender (Ref=Female) & $1.459(0.479-4.444)$ & 0.506 \\
\hline Mental Confusion (Ref:Absent) & $1.076(0.325-3.559)$ & 0.905 \\
\hline Liver Metastases (Ref:Absent) & $0.957(0.260-3.520)$ & 0.947 \\
\hline Lung Metastases (Ref:Absent) & $0.500(0.148-1.687)$ & 0.264 \\
\hline Bone Metastases (Ref:Absent) & $0.881(0.294-2.633)$ & 0.820 \\
\hline LMC ECOG PS & & \\
\hline$<2$ (Ref) & 1 & - \\
\hline$\geq 2$ & $2.976(0.602-$ & 0.181 \\
\hline Paranchymal Mass (Ref:Absent) & $14.718)$ & \\
\hline LMC Treatment & $0.858(0.262-2.810)$ & 0.801 \\
\hline No treatment (Ref) & 1 & - \\
\hline RT & $0.429(0.079-2.339)$ & 0.328 \\
\hline RT+Intrathecal MTX & $0.429(0.073-2.512)$ & 0.348 \\
\hline RT Type & & \\
\hline Cranio-spinal (Ref) & 1 & - \\
\hline Cranial & $0.846(0.283-2.530)$ & 0.765 \\
\hline TPM & 1 & - \\
\hline$\leq 6$ (Ref) & $1.458(0.484-4.392)$ & 0.502 \\
\hline$>6$ & & \\
\hline TPM: time from primary tumor diagnosis to LMC diagnosis &
\end{tabular}

\section{Discussion}

In recent studies, $m$ OS after LMC diagnosis ranged from 0.7 months to 4.8 months [9-14], in our study m OS was 1.5 months which was similar to literature. Clinical presentation with cerebral symptoms were the most common symptoms in conformity with the literature $[7,12,14]$. But, in our study nature of the clinical symptoms were not found to have prognostic effect as Giglio et al's study [2].

Whereas, LMC was mostly seen in breast cancer patients in Western countries [7,9,10,12,14], Asian studies reported that LMC occurred most frequently in lung cancer patients $[11,13]$. Consistent with Asian studies, in our study, lung cancer was the most frequent type of cancer and breast cancer was the second most common one seen in LMC patients. In order to find out the ethnic differences in LMC, more population-based studies are needed worldwide.

LMC is a progressive disease that is resistant to current therapeutic options. What is important in LMC is to predict which patient will benefit from intensive treatment. Hyun et al., reported age under 55, female gender, high performance status and active treatment with RT and IT chemotherapy were favorable prognostic factors with $\mathrm{m}$ OS of 3 months (95\% CI 2.7-3.3) [4]. Unlike this research, we did not find any association between survival and gender, ECOG PS, treatment types. Boogerd et al., searched IT chemotherapy benefit in breast cancer patients with LMC [15]. They concluded that addition of IT chemotherapy to standard cytotoxic treatment with involved field RT did not improve survival (median survival of IT patients was 18.3 weeks and 30.3 weeks for non-IT patients $(\mathrm{p}=0.32))$ or neurological symptoms. Our study also showed no difference with the implication of IT MTX. On the contrary, Lee et al., reported favorable survival with the application of IT chemotherapy ( 17 weeks versus 8 weeks, $p<0.001$ ) [1]. Another two studies showed a trend in increased survival with the application of treatment regardless of the treatment type $[2,4]$.

El Shafie et al., reported that patients with good clinical performance showed an improved m OS of 28.3 weeks, whereas, patients with poor clinical performance showed a m OS of 9.3 weeks. $(p<0.001)$ [16]. Patients with good clinical performance also showed improvement in neurological functional scale as prognostic factor for superior OS. Another report by El Shafie et al., also showed that there was no prognostic significance of primary tumor histology on OS (including 39\% breast cancer, 28\% lung cancer, $8 \%$ gastrointestinal cancer, $6 \%$ malign melanoma, $5.5 \%$ prostat cancer) [17]. They showed that RT fields (cranial, cranio-spinal or spinal) did not influence the OS which was similar to our results.

While some previous studies did not find age as a prognostic factor like our study, some other studies found older age as a negative prognostic factor $[2,4,6,15]$. Good ECOG PS was found as a positive prognostic factor in many studies $[1,4,6,7,13]$ whereas another study [2] had found no association between survival and ECOG PS like our study.

Time from primary tumor diagnosis to LMC (TPM) $>67$ weeks was reported to be independently associated with longer OS regardless of treatment type [7] but Giglio et al. [2] and Gwak et al. [6] found no association between survival and TPM like our study. In our study TPM was dichotomized by median value of 6 months and was not found as a prognostic factor.

Limitations of the current study were its retrospective nature which may cause selection bias owing to patients with poor performance and older age as they did not get any treatment. Due to retrospectivity we could not record neurological functional scale. In previous studies $21-31 \%$ of patients were reported to be LMC positive on magnetic resonance images only $[4,12,18]$ but in our study only cytologically proven patients with LMC were included. 
This limitation resulted in small number of patients being included in the study.

\section{Conclusions}

Overall survival after LMC diagnosis is too short to see the effects of treatment. Our study did not find any favorable or unfavorable prognostic factor on survival after LMC diagnosis. Initially, the nature of this rare disease must be investigated, though it is difficult to define the risk factors, the treatment must be guided accordingly. We believe that the right diagnosis leads to the appropriate treatment option for each individual patient.

Conflict of interest: None declared by the authors.

\section{References}

1. Lee SJ, Lee JI, Nam DH, et al. Leptomeningeal carcinomatosis in non-small-cell lung cancer patients: impact on survival and correlated prognostic factors. J Thorac Oncol 2013; 8:185-91. doi: 10.1097/JTO.0b013e3182773f21.

2. Giglio P, Weinberg JS, Forman AD, Wolff R, Groves MD. Neoplastic meningitis in patients with adenocarcinoma of the gastrointestinal tract. Cancer 2005; 103: 2355-62.

3. Kak M, Nanda R, Ramsdale EE, Lukas RV. Treatment of leptomeningeal carcinomatosis: current challenges and future opportunities. J Clin Neurosci 2015; 22:632-7. doi: 10.1016/j.jocn.2014.10.022.

4. Hyun JW, Jeong IH, Joung A, Cho HJ, Kim SH, Kim HJ. Leptomeningeal metastasis: Clinical experience of 519 cases. Eur J Cancer 2016; 56:107-4. doi: 10.1016/j.ejca.2015.12.021

5. Smalley KS, Fedorenko IV, Kenchappa RS, Sahebjam S, Forsyth PA. Managing leptomeningeal melanoma metastases in the era of immune and targeted therapy. Int J Cancer 2016; 139:1195-201.

6. Gwak HS, Joo J, Kim S, et al. Analysis of treatment outcomes of intraventricular chemotherapy in 105 patients for leptomeningeal carcinomatosis from non-small-cell lung cancer. J Thorac Oncol 2013; 8: 599-605. doi: 10.1002/ ijc.30147.

7. Palma JA, Fernandez-Torron R, Esteve-Belloch $\mathrm{P}$, et al. Leptomeningeal carcinomatosis: prognostic value of clinical, cerebrospinal fluid, and neuroimaging features. Clin Neurol Neurosurg 2013; 115:19-25.

8. Segura PP, Gil M, Balañá $C$, et al. Phase II trial of temozolomide for leptomeningeal metastases in patients with solid tumors. J Neurooncol 2012;109:137-42. doi:10.1016/j. clineuro.2012.03.048.

9. Herrlinger U, Forschler H, Kuker W, et al. Leptomeningeal metastasis: survival and prognostic factors in 155 patients. JNeurol Sci 2004; 223:167e78.

10. Bruna J, González L, Miró J, Velasco R, Gil M, Tortosa A. Leptomeningeal carcinomatosis:prognostic implications of clinical and cerebrospinal fluid features. Cancer 2009; 115:381-9. doi: 10.1002/cncr.24041.

11. Waki F, Ando M, Takashima A, et al. Prognostic factors and clinical outcomes in patients with leptomeningeal metastasis from solid tumors. J Neurooncol 2009; 93:205-12. doi: 10.1007/s11060.008.9758-3.

12. Clarke JL, Perez HR, Jacks LM, Panageas KS, Deangelis LM. Leptomeningeal metastases in the MRI era. Neurology 2010;74:1449-54. doi:10.1212/WNL.0b013e3181dc1a69.

13. Du C, Hong R, Shi Y, Yu X, Wang J. Leptomeningeal metastasis from solid tumors: a single center experience in Chinese patients. J Neurooncol 2013; 115: 285-91.doi: 10.1007/s11060.013.1228-x.

14. Passarin MG, Sava T, Furlanetto J, et al. Leptomeningeal metastasis from solid tumors: a diagnostic Leptomeningeal metastasis from solid tumors: a diagnostic and therapeutic challenge. Neurol Sci 2015; 36:117-23. doi: 10.1007/ s10072.014.1881-7.

15. Boogerd W, van den Bent MJ, Koehler PJ, et al. The relevance of intraventricular chemotherapy for leptomeningeal metastasis in breast cancer: a randomised study. Eur J Cancer 2004;40:2726-33.

16. El Shafie RA, Böhm K, Weber D, et al. Outcome and prognostic factors following palliative craniospinal irradiation for leptomeningeal carcinomatosis. Cancer Manag Res 2019;11:789-801. doi: 10.2147/CMAR.S182154.

17. El Shafie RA, Böhm K, Weber D, et al. Palliative radiotherapy for leptomeningeal carcinomatosis-analysis of outcome, prognostic factors, and symptom response. Front Oncol 2019;8:641. doi: 10.3389/fonc.2018.00641.

18. Freilich RJ, Krol G, DeAngelis LM. Neuroimaging and cerebrospinal fluid cytology in the diagnosis of leptomeningeal metastasis. Ann Neurol 1995;38:51-7. 